\title{
Le problème de la typologie des discours
}

Introduction et bibliographie sur les deux documents de Vossius

\section{Francis Goyet}

\section{(2) OpenEdition}

\section{Journals}

Édition électronique

URL : http://journals.openedition.org/rhetorique/122

DOI : $10.4000 /$ rhetorique. 122

ISSN : 2270-6909

Éditeur

UGA Éditions/Université Grenoble Alpes

Édition imprimée

ISBN : 978-2-84310-255-4

Référence électronique

Francis Goyet, "Le problème de la typologie des discours », Exercices de rhétorique [En ligne], 1 | 2013, mis en ligne le 12 novembre 2013, consulté le 12 septembre 2020. URL : http://

journals.openedition.org/rhetorique/122 ; DOI : https://doi.org/10.4000/rhetorique.122

Ce document a été généré automatiquement le 12 septembre 2020.

\section{(c) (i) (2)(2)}

Les contenus de la revue Exercices de rhétorique sont mis à disposition selon les termes de la Licence Creative Commons Attribution - Pas d'Utilisation Commerciale - Partage dans les Mêmes Conditions 4.0 International. 


\section{Le problème de la typologie des discours}

Introduction et bibliographie sur les deux documents de Vossius

Francis Goyet

1 Dans ce premier numéro, nous commençons l'édition des douze chapitres que Vossius consacre à ce que nous nommerons des types de discours et que son éditeur en 1660 appelle « certains genres particuliers de discours ». Plutôt que de suivre l'ordre de ses chapitres, nous allons d'emblée à la conciliatio et à son pendant la concitatio. Dans le numéro suivant, nous passerons à un discours encore plus méconnu, l'expostulatio.

2 Cicéron mentionne quelques-uns de ces types de discours, affirme qu'ils nécessitent autant d'éloquence que plaidoiries et plaidoyers au Forum, mais ajoute qu'aucun traité n'en donne les préceptes. Vossius et son époque ont donc cherché à compléter Cicéron. Sans ce complément, l'application de la théorie à la pratique est une gageure. Ce l'était déjà du temps de Vossius, ce l'est encore plus aujourd'hui. Comme nous ne lisons plus guère ce que Cicéron ou Quintilien décrivent en priorité, et que leurs traités ne décrivent pas ce que nous lisons, l'impasse est presque totale.

\section{Introduction générale à la typologie des discours}

3 L'étude des types de discours est donc un complément, mais un complément très nécessaire et, à l'époque de Vossius, très attendu. Il nous semble que l'attente est la même aujourd'hui, pour des raisons similaires.

4 Chez Cicéron et Quintilien, ce qui est massivement étudié est la plaidoirie d'avocat. Il suffit d'ouvrir un historien comme Tite-Live pour voir surgir les autres types de discours, là aussi de façon massive. La plaidoirie n'est plus alors qu'un type parmi bien d'autres - on songe immédiatement à la harangue aux troupes avant la bataille. Le résultat est que l'on ne peut pas demander à Quintilien le descriptif de la harangue. Les lecteurs des $\mathrm{XVI}^{\mathrm{e}}$ et $\mathrm{XVII}$ e siècles n'ont pu qu'être sensibles à ce manque, pour trois raisons : ils lisent beaucoup plus les historiens que nous, ils accordent une bien plus 
grande attention à leurs discours insérés, enfin ils pratiquent à grande échelle et de façon constante l'analyse rhétorique détaillée.

5 Pour répondre à l'attente de ces lecteurs-là, la première solution fut de donner des tableaux récapitulatifs. En 1545, quand Joachim Perion publie un recueil de la totalité des discours de Tite-Live, il donne pour chacun une brève analyse rhétorique et ajoute un classement d'ensemble par types. En 1570, Henri Estienne généralise ce modèle à l'ensemble des historiens de l'Antiquité, avec son fameux Conciones ou recueil de discours publics, en latin concio. Mais on n'a pas encore là de descriptif théorique pour chaque type. Il nous semble que Vossius est le premier à en donner un, au début du $\mathrm{XVII}^{\mathrm{e}}$ siècle. Son geste théorique est là aussi une généralisation. Sa liste en tant que telle n'est pas une nouveauté : le Xvi e siècle, à partir d'Érasme, l'a ressuscitée de l'Antique, mais en la traitant uniquement à propos de l'art épistolaire. Vossius a sous la main Melchior Junius, le successeur de Sturm à Strasbourg. Junius dans son traité d'épistolaire donne pour chaque type de lettre un descriptif théorique fouillé, avec pour exemples les seules Familiares de Cicéron, synthétisant ainsi le travail de Melanchthon qui avait analysé la totalité des mêmes Familiares. Le geste de Vossius est d'appliquer cet acquis du siècle précédent aux historiens. Cela revient à rapprocher les deux sortes de traités de l'Antiquité détaillant des types de discours, ceux de Ménandre le rhéteur (oraison funèbre, épithalame, " discours d'ambassade ", etc.) et les traités d'épistolaire des Pseudo-Libanios (quarante-et-un types de lettres) et PseudoDémétrios (vingt-et-un). Mais parce qu'il réfléchit à partir des historiens, Vossius ouvre considérablement la perspective. Il est lui-même obligé de faire évoluer la théorie, comme on le verra dès ce numéro avec ses deux descriptifs successifs du discours de conciliatio.

6 Ce n'est pas un hasard si les $\mathrm{XVI}^{\mathrm{e}}$ et XVII ${ }^{\mathrm{e}}$ siècles nous ramènent ainsi à la "seconde sophistique ", cette rhétorique du temps de l'empire romain dont l'étude est en plein essor ${ }^{1}$. L'enjeu est pour nous, aujourd'hui, très important. Le renouveau de la rhétorique à partir des années 1960 nous avait fait miroiter l'application de la théorie à nombre de discours; c'était déjà la même promesse au Xvi ${ }^{e}$ siècle. Mais, tout comme cette époque, nous avons fini par nous rendre compte que cette promesse est intenable quand on se réfère aux seuls grands traités, centrés qu'ils sont eux-mêmes sur les seuls grands types, plaidoiries et en général discours d'assemblée. L'intérêt d'étudier les autres types de discours n'est donc pas seulement de compléter notre information. Il est de pouvoir enfin appliquer la rhétorique, de façon satisfaisante, au texte littéraire qui est aujourd'hui notre objet propre, et en particulier au texte littéraire de l'âge classique. Dans les œuvres que nous étudions, de L'Énéide à La Thébaïde, c'est-à-dire de l'épopée au théâtre, en passant par le roman, le recueil de lettres ou de poèmes, ce qui est rare ce sont les plaidoiries, alors que les autres types de discours sont bien plus fréquents, exhortation, consolation, réclamation, etc. Les Odes d'Horace sont par exemple pour leur commentateur Minturno "action de grâces [remerciement, lat. actio gratiarum], invective, blâme, reproche ${ }^{2}$ ».

7 Il est temps d'explorer cette voie de façon un peu systématique. Mais ce ne sera pas facile, comme va l'expliquer cette introduction générale, et pour des raisons de fond, liées à l'idée même de type. Dans un premier point nous décrirons un cas exemplaire, chez Cicéron lui-même, pour faire saisir ce que signifie reconnaître une forme ou un type. Nous donnerons ensuite un aperçu d'ensemble de la liste de Vossius, qui est une bonne représentante des listes assez variées que l'on trouve à son époque. Nous 
reviendrons enfin sur l'idée de typologie. Le type n'est pas une norme, et c'est bien pour cela qu'il y a à la fois stabilité et variation. Il est donc difficile et néanmoins possible, à force d'entraînement, de reconnaître le type ou en latin la forma.

\section{Un exemple chez Cicéron : l'exhortation}

8 Cicéron n'ignore rien des types de discours autres que la plaidoirie ou le grand discours devant une assemblée politique, même si, nous l'avons dit, il relève que de ces autres types il n'y a ni traités ni préceptes3. Dans son De l'orateur, il évoque "les genres et espèces de discussion " (III, 119, generibus ac modis disceptationibus). Parlant du genre de " celles qui se rapportent à la pratique, ad agendum », Cicéron en nomme quatre souscatégories, "l'exhortation, l'objurgation, la consolation, l'appel à la pitié»: cohortationes, objurgationes, consolationes, miserationes $\left(\$ 118^{4}\right)$. Il est frappant de voir qu'en 1767, Jean-Baptiste Crevier a pour le genre délibératif une liste réduite elle aussi à quatre termes, très proches : " Exhorter, reprendre, demander, consoler » (p. 21 de sa Rhétorique..., voir la Bibliographie tout à la fin de ce fichier). Nous pouvons donc pour une première réflexion partir de cette liste très abrégée, et nous en tenir même à son premier item, l'exhortation, qui a quelque chose d'emblématique. Nous le pouvons d'autant plus qu'au début de son ouvrage, Cicéron donne un bel exemple d'exhortation, et qu'un peu auparavant, il a annoncé cet exemple par une sorte d'analyse théorique. Nous allons ainsi nous placer à ce moment originel du processus qui mène à reconnaitre un type de discours. Sans ce premier repérage, rien ne peut s'enclencher.

L'exemple d'exhortation dans De l'orateur est l'espèce de tirade par laquelle Crassus lance le dialogue en encourageant les nobles jeunes gens qui l'écoutent à devenir, à son égal, de très grands orateurs (I, 30-34, avec cohortandum au tout début). Cette exhortation est d'abord, d'un point de vue presque extérieur, un petit discours en forme, et pas un flux de paroles lancées au hasard. En effet, Crassus termine sa tirade en disant « je conclurai en peu de mots» ( $\$ 34$, comprendam brevi). Du coup, le lecteur qui n'aurait pas repéré comme marqueur le cohortandum initial revient sur ses pas. S'il y a à la fin une conclusion, c'est un indice que le début pourrait être un exorde. Voici ce début dans la traduction Courbaud ${ }^{5}$ : "Crassus amena la conversation sur le sujet de l'éloquence. 30. Il commença par déclarer que Sulpicius et Cotta lui paraissaient moins à exhorter [cohortandum] qu'à féliciter [conlaudandum], puisque leur talent les avait non seulement... mais encore... » Une fois qu'on a compris que l'ensemble de la tirade est un discours, on comprend que ce petit éloge initial était en fait l'éloge du destinataire qui est de règle dans un exorde. On remarque aussi que le "Il commença par déclarer » rend exorsus, sur exordior. Il faudrait donc traduire: «Son exorde fut que Sulpicius et Cotta... »

10 Suit, au style direct, le discours proprement dit, dont voici le résumé. Premier argument : l'éloquence est l'art qui a toujours dominé, du moins dans une Cité en paix, et digne de ce nom (\$30). Deuxième argument, très développé : seul face à la multitude, l'orateur a un rôle presque « royal » (\$ 31-32). Troisième argument, en apparence plus faible: même loin du Forum, la parole dans la conversation est ce qu'il y a de plus agréable, de plus humain (fin du § 32 ). «Et puis, venons-en au point capital [ad illa summa]». Ce dernier argument articule les précédents de façon grandiose: c'est à l'éloquence que l'on doit la civilisation et le droit, c'est elle qui nous rend véritablement humains, sinon nous vivrions encore comme des bêtes sauvages (§33). Conclusion : du 
grand orateur dépend «non seulement sa dignitas à lui mais le salut même de tous, et de l'État tout entier "; donc, jeunes gens, embrassez avec ardeur cette carrière, à la fois glorieuse pour vous et utile aux autres, vos amis comme l'État, « et vobis honori et amicis utilitati et rei publicae emolumento " ( $\$ 34$, notre traduction).

11 Sur cette chute s'achève ce petit discours d'à peine deux pages. L'ensemble est l'équivalent d'une tirade de théâtre: quand Crassus a conclu, un autre personnage prend la parole, pour le contredire. En d'autres termes, ce discours est autonome, il a son unité. L'éloge même de l'éloquence n'y est pas une fin en soi, elle sert le but du discours, exhorter de nobles jeunes gens à devenir des orateurs.

Venons-en maintenant à l'analyse théorique. À partir du moment en effet où on a repéré que c'est là un discours autonome, et qui a une forme, la question se pose de décrire cette forme en tant que telle. Avant la tirade de Crassus, le narrateur du dialogue, Cicéron lui-même, a donné des indications en fait théoriques (I, 19): "Exhortons [hortemur] nos enfants, et tous ceux dont la gloire et les succès [gloria et dignitas] nous sont chers, à se bien pénétrer de la grandeur de cet art [rei magnitudinem ]» - litt. " de la grandeur de la chose », la chose ou "art » étant la rhétorique. La traduction Courbaud, de nouveau, gomme ce que la formule rei magnitudo a de standard et de technique. Ce latin est du jargon. Il signale un motif ou «lieu» récurrent, commun à plusieurs types de discours : l'importance de l'enjeu. Un autre motif, tout aussi classique, est désigné de façon moins technique par gloria et dignitas : c'est l' honestas ou honorable. On est ainsi sur la piste du couple habituel des deux lieux du délibératif, « l'utile et l'honnête ». La chute de l'exhortation de Crassus le confirme : « et vobis honori et amicis utilitati ». Soit $a$ ) l'honnête et b) l'utile : a) grandeur de votre rang quasi «royal», donc gloire maximale, pour vous; b) grandeur de l'enjeu, utilité également maximale pour vos amis et pour la Cité en tant que telle. La grandeur ou rei magnitudo désignait donc en fait l'ensemble utile + honnête. La rhétorique est de première importance, de quelque côté qu'on l'envisage, s'il n'y a pas de carrière plus noble et plus profitable, pour vous comme pour l'État ${ }^{6}$.

13 Nous sommes encore loin des indications théoriques de Vossius sur l'exhortation. Mais ce bref descriptif a suffi à notre propos, en nous permettant de retrouver les deux étapes initiales de toute reconnaissance de forme, celles d'où découle la suite et dont ne parle pas Vossius, tant cela lui paraît évident.

14 La première étape est de mettre un nom sur la chose. Le simple fait de voir et de nommer « exhortation » la tirade de Crassus est un gain en compréhension, un gain capital. En équipe, nous l'avions senti avec les titres d'un de nos commentateurs ${ }^{7}$. «<Oratio > Aeneae extinctum Pallantem lugentis » (Énéide, XI, 42-58) : nous avions d'abord traduit «<Discours> d'Énée se lamentant sur le corps de Pallas». Mais c'est tout autre chose de dire : "déploration (luctus ${ }^{8}$ )", ou " oraison funèbre de Pallas ». On ne regarde plus ce que dit Énée comme un flux informe de paroles, dictées par la seule douleur, mais comme un discours en forme, et analysable en tant que tel. Il en va de même pour "Qua Aeneas ex Epiro discedens Helenum, et Andromacham alloquitur» (Aen. III, 493-505). Non pas "Énée en partant d'Épire adresse quelques mots à Hélénus et Andromaque ", mais «allocution de départ ». Une allocution, c'est un type de discours, et cela change tout. Vossius nous l'a confirmé, puisqu'il consacre à ce type de discours un chapitre entier de sa rhétorique abrégée (II, 20), et qu'il l'identifie, au titre, par le même discedens, lui-même repris de Scaliger ${ }^{9}$. Ce discedens apparemment banal était donc en fait le marqueur d'un type de discours, ajouté par Scaliger à la liste qu'il trouve chez 
Ménandre le rhéteur - l'allocution de départ étant le symétrique du discours de bienvenue. En mettant ainsi un nom sur la chose, le substantif est le premier pas indispensable à une reconnaissance des formes, c'est la condition sine qua non de toute la suite.

L'étape suivante est de confronter la forme identifiée à un type déjà reconnu, à une typologie. Du côté de la matière, il s'agit de savoir quels sont les divers «lieux » que peut ou même doit convoquer ce type particulier de discours; et, du côté de la forme, quel plan il peut ou doit suivre. Après avoir mis un nom sur la chose, il faut donc aller du côté de la théorie, et de là on reviendra au texte. Par exemple, dans le cas du discours de Crassus : y a-t-il des lieux de l'exhortation? Ceux auxquels recourt Cicéron sont-ils standard ou non standard? En trouve-t-on d'autres chez Vossius, mais aussi chez les autres auteurs de traités (la réponse est oui) ? L'orateur doit-il les utiliser en tout ou partie? Et dans quel ordre ? C'est à cette sorte d'enquête ou de questionnement qu'invite notre publication, une enquête qui peut d'ailleurs mener très loin.

\section{Aperçu d'ensemble de la liste de Vossius}

Pour l'instant, nous n'avons aperçu qu'une liste très brève de quatre items, « exhorter, reprendre, demander, consoler ». Nous pouvons maintenant regarder la liste complète, au moins dans ses grandes lignes, et en nous limitant à la Rhétorique abrégée de Vossius $^{10}$.

Ses livres I et II traitent la première partie de la rhétorique, l'inventio. L'invention est subdivisée en generalis et specialis. La générale couvre le livre I et la première moitié du livre II. Le livre I traite les arguments proprement dits, ceux du logos (de la raison ou docere), et le livre II, les autres « arguments » (argumenta), ceux des affectus (II, 1), c'està-dire les lieux du pathos ou movere (chap. 2-14) et de l'ethos ou conciliare (chap. 15). La suite et fin du livre II (chap. 16-27) est la partie qui nous intéresse. Vossius l'intitule, au titre de son chapitre 16, une partie spéciale ou spécifique de l'inventio, «De Inventionis Parte Speciali ». Son éditeur en 1660 ajoute les accolades que voici, très parlantes. La liste des types de discours est en page de droite ${ }^{11}$ : 


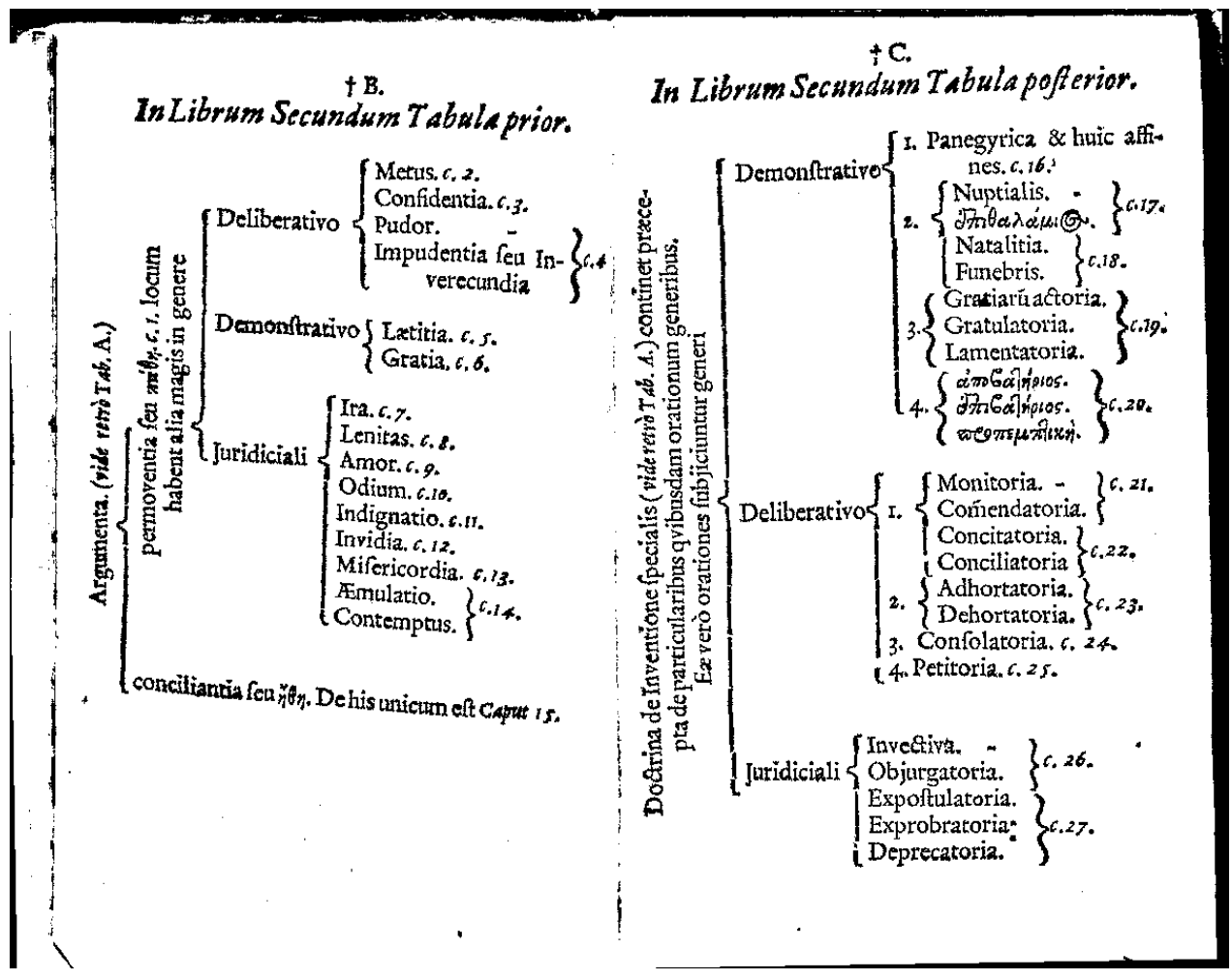

En page de droite, les types de discours, du chap. 16 au chap. 27

Collection privée. qui « ont de l'affinité » avec le panégyrique et ne sont pas nommés.

Voici cette liste en français, en ne citant que les discours les plus connus :

-Genre épidictique, 4 sous-catégories (dites en latin classes, II, 16, §3), pour 11 types de discours :

1. le panégyrique « et les discours qui ont de l'affinité » (II, chap. 16);

2. l'épithalame (chap. 17) et l'oraison funèbre (chap. 18);

3. le discours de remerciement (chap. 19);

4. le discours de bienvenue, de départ (l'apobatèrios), etc. (chap. 20).

- Genre délibératif, 4 sous-catégories, pour 8 types de discours :

1. l'admonestation (chap. 21), les discours de conciliatio et concitatio (chap. 22);

2. l'exhortation (chap. 23) ;

3. la consolation (chap. 24);

4. la « pétition » ou discours de requête (chap. 25).

- Genre judiciaire. Pas de sous-catégories numérotées, mais une simple liste de 5 types: invective et objurgation (chap. 26);

expostulatio, reproches et déprécation (chap. 27).

Pour le délibératif, on note que les quatre sous-catégories de Vossius sont les quatre de Crevier, "exhorter, reprendre, demander, consoler", si du moins l'on admet que demander désigne la petitio et reprendre (reprehensio), l'admonestation ou monitio.

C'est dire que la liste de Vossius est une des nombreuses variantes d'une liste plus ou moins standard qui circule du $\mathrm{XvI}^{\mathrm{e}}$ au XvIII ${ }^{\mathrm{e}}$ siècle, et qui a comme fusionné les listes 
héritées de la seconde sophistique. En voici deux bornes significatives. En 1521, dans son Art d'écrire des lettres, Érasme donne, pour le délibératif : "Conciliatio, reconciliatio; exhortatio, dehortatio ; suasio, dissuasio ; consolatio, petitio, commendatio, monitio, amatoria »; et pour le judiciaire: "Accusatio, querela, defensio, expostulatio, expurgatio, exprobratio, comminatio, invectiva, deprecatio ${ }^{12} »$. En 1747, dans son Cours de belles-lettres, Charles Batteux introduit ainsi le « Style Épistolaire » (p. 271):

Le Genre épistolaire n'est autre chose que le genre oratoire rabaissé jusqu'au simple entretien; par conséquent il y a autant d'espèces de lettres qu'il y a de genres d'oraisons [c'est-à-dire de discours, orationes].

On conseille dans une lettre, on détourne, on exhorte, on console, on demande, on recommande, on réconcilie, on discute : et alors on est dans le genre délibératif. On accuse, on se plaint, on menace, on demande que les torts soient réparés : c'est le genre judiciaire. On loue, on blâme, on raconte, on félicite, on remercie, etc. : c'est le genre démonstratif.

D'Érasme à Batteux, il est significatif de voir que la liste a particulièrement bien survécu dans l'épistolaire ${ }^{13}$. Tout notre propos est au fond de la sortir de cet espace un peu confiné, c'est-à-dire de répéter le geste de Vossius : la transférer des lettres aux discours. L'opération est facilitée par le féminin des adjectifs, petitoria, monitoria, etc., sous-entendu oratio ou epistola. Une " pétitoire » ou une "monitoire », cela peut-être autant un discours qu'une lettre de requête ou d'admonestation ${ }^{14}$. Les deux traités de Vossius donnent comme exemples, pour chacune de leurs catégories, des lettres mais aussi, et surtout, des discours ; il en va de même, à la même époque, dans le traité de Tesmar. La liste plus ou moins standard ignore les frontières entre discours fictifs et discours réels, comme d'ailleurs entre prose et poésie.

Pour revenir à la liste de Vossius, et conclure là-dessus, l'éditeur avec ses accolades suggère un rapprochement avec l'autre liste, en page de gauche. C'est celle des passions, reprises d'Aristote, et traitées aussi dans le second livre de Vossius :

- délibératif : metus, confidentia, pudor, impudentia (pour les passions, chap. 2-4; pour les types de discours, chap. 21-25) ;

-épidictique : laetitia, gratia (chap. 5-6; chap.16-20) - la laetitia n'est pas dans la liste d'Aristote ;

- judiciaire : ira, lenitas, amor, odium, indignatio, invidia, misericordia, aemulatio liée à contemptus (chap. 7-14; chap. 26-27).

Même si Vossius ne fait pas explicitement le lien, celui-ci est suggéré par ses définitions pour chaque type de discours. Ainsi, dans le genre délibératif, on a, pour le couple exhortation et " déhortation" (l'exhortation à ne pas faire) : «ici est surtout suscitée l'assurance, ou au contraire la crainte, fiducia, aut metus »-la fiducia est dans les accolades la confidentia. La correspondance est moins nette dans le cas de l'admonestation et types liés, qui relèvent du délibératif mais où « dominent colère, haine, ou amitié, affectus ira, odii, vel amoris ", passions classées dans le judiciaire - la lenitas ici omise est le pendant implicite de l'ira. Nous n'avons pas de solution pour ce petit problème. Notons simplement qu'il nous ramène aussitôt au chapitre 22 sur la conciliatio, que nous donnons ensuite. Là où le discours de concitatio suscite l'émeute ou sédition (ira), son inverse la conciliatio vise l'apaisement (lenitas) : c'est chez Aristote le balancement entre les chapitres 2 et 3 de la Rhétorique, II.

Ce flottement relatif signale la difficulté inhérente à l'analyse, difficulté que ne doivent pas masquer les belles répartitions par accolades. Autant le grand discours d'assemblée est le modèle ou type par excellence, avec son plan canonique, autant pour les autres 
types de discours c'est "moins simple ", comme le dit d'emblée Vossius ${ }^{15}$. Rien n'est simple, pour une raison de fond, qui est qu'il ne faut pas confondre type et norme.

\section{Le type n'est pas une norme}

Les choses seraient simples, et caricaturales, si tout cela relevait du normatif. Il suffit de songer au domaine de l'épistolaire, qui est celui où la liste s'est le mieux maintenue. Quand Érasme dit qu'il va donner des literarum formae, nous comprenons : des modèles de lettres ${ }^{16}$. Ce n'est pas faux. L'idée de norme et de code est vite à l'horizon quand les lettres sont en fait des lettres d'affaires ou de diplomates. Au sein de mondes aussi contraints que l'étaient les Cours d'Europe ou la Curie romaine, il est impératif de se conformer à des modèles standard, ce qui ne va pas sans la récurrence de véritables clichés. Nous pouvons alors projeter sans peine sur le passé ce à quoi ressemble l'épistolaire quotidien d'aujourd'hui, qui enseigne comment écrire à son percepteur, à son député, comment rédiger une lettre de recommandation, etc. Le modèle ou forma menace toujours de produire du formaté ; et le type, du stéréotypé.

Mais tout n'est pas si simple. Sur le fond, le type n'est pas plus une norme que la règle n'est la loi. En le montrant, nous allons ainsi justifier notre emploi du mot type, qui n'est pas seulement un pis-aller pour remplacer des formules devenues vagues en français moderne, comme « espèces » chez Crevier (p. 20) ou « espèces de lettres » chez Batteux.

Ce n'est pas simple parce que, d'abord, et pour citer de nouveau Crevier (p. 21), « les différents genres se confondent souvent dans un seul et même discours. Le Prédicateur qui loue un Saint, nous exhorte à l'imiter. L'Orateur qui console de la mort d'un ami, loue celui dont il déplore la perte." Panégyrique, ou exhortation? Consolation, ou oraison funèbre? La solution de Crevier est élégante, mais demande elle-même du doigté : «On détermine la dénomination du discours par la partie qui y domine, et qui en fait le principal objet. »

Ensuite, d'un traité à un autre, les variations sont très nombreuses. Le même type de discours n'est pas toujours classé de même : Vossius range l'invective dans le judiciaire, tout en signalant que certains la mettent dans l'épidictique, en tant que blâme à outrance (vituperatio). Et la même dénomination à son tour peut désigner des types de discours sensiblement différents. Il suffit de comparer les divers classements des multiples Conciones pour voir nombre de différences de l'un à l'autre.

En effet, même quand la dénomination est stable, un discours donné peut être considéré par le commentateur comme relevant de tel type ou au contraire de tel autre. La conciliatio nous en fournit encore un exemple. Le discours de Tacite (Ann. I, 42-43) que Pelletier qualifie ainsi en 1641 est vu en 1785, par Gourdin, comme un discours de reproches ou exprobratio, ce qui est d'ailleurs justifié par le exprobrari de Tacite luimême (début de son $\$ 44$ ). Nous retrouvons, mais comme difficulté, la solution de Crevier. Il n'est pas simple de déterminer ce qui fait « le principal objet» d'un discours, tout dépend de la finalité qu'on lui assigne. En tout cas, baptiser un discours, c'est déjà en soi un résultat, le produit d'une réflexion.

31 Vu de plus près, le bel édifice de classification semble donc se fissurer de tous les côtés. Chaque traité ou classement est par définition bien rangé, mais il se pourrait qu'en en comparant plusieurs nous nous retrouvions en fait devant un véritable bazar. Ce serait une objection considérable. Même si dans un bazar chacun trouve son bonheur, autant 
arrêter une telle enquête avant de la commencer. La réponse à l'objection est à chercher dans l'idée philosophique de type, qui est l'autre sens du latin forma. Certes, les descriptifs de Vossius visent le systématique, mais ils ne sont pas pour autant à prendre comme du prescriptif, du normatif. Ce n'est pas un code obligé.

Forma en latin synthétise tout un pan immense de la réflexion grecque, que l'on peut schématiser ainsi. Dans une typologie, les règles sont moins des contraintes que des régularités. Le mot forma rend chez Cicéron l'Idée platonicienne mais aussi la morphè du couple aristotélicien de la matière et de la forme. Derrière type, c'est encore du grec, tupos, mot qu'emploient Épicure pour répondre à la théorie des Idées, et les Pères de l'Église pour parler du Christ. Chez Cicéron, la forme se manifeste de façon visible dans le «plan de l'ouvrage » dressé par l'artiste architecte, "plan » étant un sens spécialisé de forma. Mais même quand le plan rend visible le projet, c'est pourtant du côté de l'invisible que demeure en réserve l'essentiel, à savoir le mystère de la conception, du type, de l'Idée. Pas plus que l'Idée, le type n'est complètement saisissable par l'intelligence. On ne peut que s'en approcher. Chaque réalisation réussie l'incarne, à sa manière, sans être pour autant aisément généralisable.

Si Érasme donne en exemple les lettres exhortatoires, monitoires, etc., qu'il trouve chez de grands auteurs ou qu'il écrit lui-même, c'est pour faire saisir le type ou Idée de chaque espèce de lettre, sa forma. Cela ne signifie pas que son lecteur doive décalquer ces exemples de façon servile. La théorie de l'imitatio enjoignait de remonter à l'Idée en passant par telle ou telle incarnation accessible aux sens, tout comme, dans la célèbre formule de saint Paul, le chrétien doit remonter à l'invisible en passant par le visible. Avant Érasme, Servius de même semble ériger un des premiers discours de l'Énéide en modèle de tous les discours de demande ou requête (petitio, "pétitoire»). Mais il emploie le verbe formare. Le verbe ne signifie pas qu'il transforme le plan de ce discours en norme, en consigne universelle et impérative. Servius croit plutôt avoir découvert chez Virgile une régularité, et lever ainsi un voile sur le mystère de la création virgilienne $^{17}$. La règle n'est pas la loi, ces deux mondes sont distincts. C'est nous qui réduisons le classicisme à un corset d'impératifs, caricature commode pour servir de repoussoir, mais erreur conceptuelle qui engendre une cascade de faux problèmes, tous de notre invention.

Ainsi compris, le mot de type ou de forma nous renvoie à l'objectif visé par notre publication. Il s'agit, par la typologie, d'habituer l'œil à reconnaître des formes extrêmement variables, mobiles, éventuellement trompeuses. La méthode est analogue à ce que l'on nomme en médecine la clinique. Si les internes hospitaliers se forment en suivant le grand clinicien dans sa tournée des malades, c'est que pour leur métier théorie et pratique sont indissociables. Seul l'œil exercé sait identifier la maladie, en reconnaître la forma, repérer que cet ensemble de symptômes est un tableau qui correspond à celle-ci plutôt qu'à celle-là. C'est une affaire d'habitude, de régularités, pas de code préétabli ou de descriptifs purement livresques. Identifier la maladie, lui donner son nom, c'est le premier pas capital. Mais ce nom n'est qu'une hypothèse, le début d'une enquête. L'étape suivante est de confirmer ou d'infirmer l'hypothèse en cherchant si on retrouve des traits standards, une configuration documentée, alors même que chaque cas est en fait singulier.

Dans ce genre d'enquête, singularité et régularité ne s'excluent pas mutuellement, pas plus que pratique et théorie. Bien au contraire, l'une appelle l'autre. Le faux problème serait de croire que le singulier se définit comme un écart par rapport aux règles, ce qui 
amène aussitôt à ériger l'écart comme une glorieuse rébellion face à une loi opprimante, et, à terme, comme la liberté face à la tyrannie. Le vrai problème est que chaque configuration est singulière, hic et nunc, et pourtant régulière. Si la solution logique du faux problème est de jeter les règles à la trappe, la solution du vrai problème est, non moins logiquement, le passage par la typologie, qui permet de définir au mieux la singularité du cas, sa configuration particulière, sa liberté dans et non pas contre les règles. Chaque cas de figure est le produit d'une géométrie variable, d'une combinatoire, et pour en rendre compte la typologie est la méthode adéquate.

L'époque moderne reste mal à l'aise face aux nombreuses codifications anciennes. D'un côté, nous sommes prompts à les traiter avec une ironie condescendante, et à moquer les clichés et stéréotypes qu'elles engendrent inévitablement. De l'autre côté, et sans voir la contradiction, nous sommes au fond déstabilisés de découvrir que ces soi-disant codes étaient loin d'être appliqués de façon systématique. Ainsi posé, le problème conduit à l'impasse. L'issue passe par une découverte du genre de celle que nous avons faite en équipe. En construisant en commun l'intérêt pour les types de discours, puis en comparant les commentateurs, nous avons dans le même mouvement déconstruit l'idée de codification ou de normes.

D'un traité ou d'un classement à l'autre, les flottements relatifs sur les types de discours ne tiennent qu'à la nature de l'objet étudié. Ils ne ruinent donc en rien la possibilité même d'une méthode pour aller vers l'identification certaine. Ce n'est impossible que pour les profanes, toujours pressés. Pour les internes, la condition essentielle est d'observer de façon critique, sous la direction d'un maître, en y consacrant beaucoup de temps et de travail, un très grand nombre de cas, certains simples et d'autres compliqués. C'est bien pour cela que l'enseignement de la rhétorique passait par le Conciones, c'est-à-dire par un recueil contenant des dizaines, des cinquantaines et plus souvent des centaines de discours, tirés en ce cas des historiens, et aussi variés que nombreux. Sans la masse, on ne peut pas acquérir l'œil du clinicien, son habitude et même habitus.

Il reste beaucoup de chemin à faire. Un des signes que nous aurons acquis l'œil et l'habitude du clinicien sera la capacité à isoler une forme autonome au sein d'un plus grand discours. Dans une symphonie ou une sonate, l'oreille cultivée repère un menuet ou un rondo. De même, il nous faut apprendre ou réapprendre à isoler dans un panégyrique une partie autonome d'exhortation (souvent conclusive, comme le rondo), ou à l'inverse, un moment d'oraison funèbre à l'intérieur d'une consolation. Ces différents types « se confondent ", car rien n'est simple : à nous de savoir les distinguer, de retrouver l'œil du rhétoricien. Tels les médecins autour d'un malade, nous pourrons de nouveau discuter et nous empoigner, pour savoir si tel cas est plutôt une conciliatio ou plutôt une exprobratio. C'est ce que nous faisons régulièrement dans l'équipe RARE, lors des séances de séminaire.

39 À la différence des malades, les textes, eux, n'en meurent pas. Ou plutôt ils revivent, ils prennent un nouveau relief. En les confrontant à un type qu'ils incarnent admirablement, la discussion fait aussi saisir leur irréductible singularité. C'est logique, et non pas paradoxal. Chaque texte reste un cas, mais au sens de configuration, et non d'écart par rapport à une supposée norme. Réduire l'exceptionnel à n'être qu'exception à la règle est une vision simpliste et pour tout dire d'incultes. Une telle vision nous barre l'accès au sens si cultivé de l'admiration qu'avait développé l'âge classique. 
le dire en deux mots, l'entreprise est donc de réidentifier des objets perdus de vue, moins repérés que la plaidoirie ou le grand discours d'assemblée. Au-delà, son but est de ressusciter une discussion critique, entre cliniciens. C'est aussi ce qui justifie de passer plutôt par un séminaire et une revue, dont la devise pourrait être : ut rhetorici disputent.

\section{BIBLIOGRAPHIE}

\section{Bibliographie indicative sur les types de discours}

Ce simple repérage signale surtout ce qui est accessible sur Internet (« Google » : Google Livres). Pour l'épistolaire de l'Antiquité comme du Xvi ${ }^{\mathrm{e}}$ siècle, voir l'importante bibliographie de Luc Vaillancourt, La lettre familière au XVI ${ }^{e}$ siècle, rhétorique humaniste de l'épistolaire, Paris, Champion, 2003 ; et, pour le XVII ${ }^{\mathrm{e}}$ siècle, de Cécile Lignereux dans L'écriture de la tendresse. Les Lettres de Madame de Sévigné, à paraître chez Garnier.

BATTEux Charles, Cours de belles-lettres, Paris, Desaint et Saillant, 1753, t. IV ; Google.

BUCHLER Johann, Sacrarum profanarumque phrasium poeticarum thesaurus, Londres, Haviland, 1624. Dans l'appendice (à partir de Z3v), liste et brève définition de 21 types de poèmes, sur le modèle de Scaliger (epithalamium, eucharisticon ou remerciements, apobaterion, etc.) ; tous exemplifiés par des poèmes latins ou anglais de l'époque dans J. W. Binns, Intellectual Culture in Elizabethan and Jacobean England. The Latin Writings of the Age, Leeds, F. Cairns, 1990, chap. 5 (« Minor poetic genres »), p. 60-80.

CAUSSIN Nicolas, Eloquentiae sacrae et humanae parallela, Paris, Chappelet, 1619, livre XIII ; Gallica. Analyse et classement de 38 discours tirés du Conciones d'Estienne.

CREVIER Jean-Baptiste, Rhétorique françoise, Paris, Saillant et Desaint, 1767, p. 16-20 ; Google.

ÉRASME, De conscribendis epistolis, dans les Opera omnia, Lugduni Batavorum (Leyde), P. Van der Aa, 1703, t. I (« LB, I »), col. 379-484; éd. J.-Cl. Margolin, dans l'éd. des Opera, Amsterdam, NorthHolland, 1971, I, t. 2, p. 309-579. Le classement de ses lettres (et de Pierre Fabri, Le grand et vrai art de pleine rhétorique, I, Gallica) est mis en tableau dans Claude La Charité, La rhétorique épistolaire de Rabelais, Québec, Nota bene, 2003, p. 245-251.

ESTIENNE Henri, Conciones sive orationes ex Graecis Latinisque historicis, Paris, H. Estienne, 1570.

GOURDIN François-Philippe, Principes généraux et raisonnés de l'art oratoire, Paris, Barbou et Nyon le jeune, 1785, p. 116-129; Google.

HURTAUT Pierre-Thomas-Nicolas, Manuale rhetorices, Paris, chez l'auteur, 1782 ( $3^{\mathrm{e}}$ éd.), p. 99-105 ; Google.

JOUVANCY Joseph, Candidatus rhetoricae, Cologne, S. Noethen, 1715, V, 6, chap. 3 « Oratiuncularum formis, et propria singularum tractatione », p. 324-329 (sur digitale-sammlungen.de) ; trad. fr. H. Ferté : L'élève de rhétorique, Paris, Hachette, 1892, « Des différentes espèces de petits discours » 
(dans André Collinot et Francine Mazière, L'exercice de la parole. Fragments d'une rhétorique jésuite, Paris, Éd. des Cendres, 1987, p. 144-147). Dix-huit types de discours, dont l'apobaterion.

JUNIUS Melchior, Epistolae ex historicis, tam veteribus, quam recentioribus secundum materias ita congestae ac digestae..., Montbéliard, Lazare Zetzner, 1595 (BnF Z-14189 ; Google). Un gros recueil de lettres tirées des historiens antiques, classées, avec analyse rhétorique, et incluant des lettres du XVI ${ }^{\mathrm{e}}$ siècle.

JUNIUS Melchior, Orationum ex historicis tam veteribus quam recentioribus..., Strasbourg, Lazare Zetzner, 1598. Un très gros Conciones, incluant des discours d'historiens du XvI siècle.

JUNIUS Melchior, Scholae Rhetoricae, de Contexendarum Epistolarum, Bâle, Conrad Waldkirch, 1587 ; chap. « De conciliatoriis epistolis » p. 113-119 : http://www.uni-mannheim.de/mateo/cera/junius1/ jpg/s113.html

LE DEIST DE вотIDOUX Jean, Conciones ou discours choisis dans Salluste, Tite-Live, Tacite et Quinte-Curce, Paris, Librairie classique et d'éducation, s. d. [1823] ; avec un classement des discours par types. LIPSE Juste, Institutio epistolica (1591), éd. G. C. Kirchmaier, Wittenberg, J. Michael, 1671 ; Google. MELANCHTHON Philippe, Argumenta et scholia in Ciceronis epistolas ad Familiares (analyse de toutes les Familiares de Cicéron), dans Opera, éd. C. G. Bretschneider et H. E. Bindseil, Halis Saxonum, C. A. Schwetzchke, éd. du Corpus Reformatorum, 1851, t. XVII, col. 13-560 ; Google, via Wikipedia, « Corpus reformatorum », puis le lien « CR 17-1851».

MÉNANDRE LE RHÉTEUR, Menander rhetor [texte des deux traités], éd. D. A. Russell et N. G. Wilson, Oxford, Oxford U. P., 1981 ; trad. M. G. Garcia et J. G. Calderon, Dos tratados de retorica epidictica, Madrid, Gredos, 1996.

PAjoT, Charles, sj, Tyrocinium eloquentiae, sive Rhetorica nova, et facilior ( $1^{\text {re }}$ éd. 1647), Cambrai, Frères Dufour, 1650 ; Google. Au livre III, trois chapitres sur les « variae orationes » : ch. III, démonstratif (p. 307-318); ch. V, délibératif (p. 330-339); ch. VIII, judiciaire (p. 349-356).

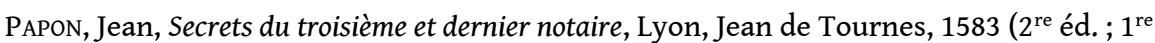
éd. 1578) ; Google. La fin du livre I de ce "Secrétaire » est consacrée aux « lettres closes » (p. 43-72) et donne, en français, la liste d'Érasme dans le De conscribendis epistolis (p. 46-47 et p. 57), et un descriptif des lettres relevant du délibératif (p. 58-68), du démonstratif (p. 68-69) et du judiciaire (p. 69).

PELLETIER Gérard, Reginae Palatium Eloquentiae, Lyon, J.-A. Candy, 1653 ( $1^{\text {re }}$ éd. Paris, Buon, 1641), punctus IX, «Artificium Conciliationis », p. 813-815 (Gallica) ; Mainz, J. G. Schönwetter, 1652, punctus IX, p. 813-815 (Google).

PERION Joachim, In omnes T. Liuii Conciones, Bâle, Rob. Winter, 1545 (sur digitale-sammlungen.de). Au total, 193 discours.

PSEUDO-LIBANIOS et PSEUDO-DÉMÉTRIOS DE PHALÈRE, Lettres pour toutes circonstances, éd. P.-L. Malosse, Paris, Les Belles Lettres, 2004. Le traité du Ps.-Libanios a eu une dizaine d'éd. européennes aux $\mathrm{XVI}^{\mathrm{e}}$ et XVII ${ }^{\mathrm{e}}$ siècles.

SCALIGER, Poetices libri septem [pages de l'éd. Lyon, Antoine Vincent, 1561], éd. L. Deitz, Stutgart, Bad Cannstat, 1995 (pour le livre III).

TESMAR Johannes, Rhetoricarum exercitationum libri VIII, Amsterdam, Elzévir, 1657 ; Google. Classement des types de discours, avec de très nombreux exemples, p. 83 sq. ; classement et analyse de tous les discours de Tite-Live (205 discours), p. 269-506. 


\section{NOTES}

1. Voir les travaux de L. Pernot et de son équipe à Strasbourg, avec des ouvrages consacrés à des types de discours, «la supplication », « la prière », «l'hymne en prose » (voir les n ${ }^{\text {os }} 10,11$ et 15 dans la collection « Recherches sur les rhétoriques religieuses», Turnhout, Brepols Publishers). Voir aussi P. Fleury, Lectures de Fronton. Un rhéteur latin à l'époque de la seconde sophistique (Paris, Les Belles Lettres, 2006), avec un chapitre sur la consolation (ibid., p. 65-99) : de ce type le rhéteur « connaît et maîtrise les règles [...] mais son propos n'est pas d'y être fidèle à tout prix » (ibid., p. 99) ; Fronton est le maître de rhétorique de Marc Aurèle.

2. N. Dauvois, La vocation lyrique. La poétique du recueil lyrique en France à la Renaissance et le modèle des Carmina d'Horace, Paris, Garnier, 2010, p. 124 (laquelle précise avoir été alertée sur les types de discours par les travaux de notre propre équipe) ; avec (p. 128-129) deux listes de titres des Odes, chez deux humanistes: "la louange joue un rôle important mais pas davantage que le conseil, l'avertissement, la prière, l'invective ", soit autant de types bien répertoriés. N. Dauvois souligne l'intérêt pour la lecture d'Horace: l'attention à ces types de discours oblige à reconsidérer entièrement la définition même du lyrisme antique.

3. Cicéron, De Oratore, II, 50 : pas « de règles particulières [certum praeceptorum genus] ", donc pas non plus d'ouvrage théorique où trouver des règles sur ces types de discours, « ex artificio res istae praecepta non quaerunt ».

4. À II, 50, la liste se résume aux trois premiers : « objurgatio, cohortatio, consolatio ». - De même que nous moderniserons dans nos citations les graphies du français, de même nous donnons le latin avec $j$ et $v$, comme le Gaffiot.

5. Paris, Les Belles Lettres, 1966, $1^{\text {re }}$ éd. 1928.

6. Voir Tesmar (p.61 de ses Exercitationes, voir la Bibliographie), transposant à l'étude de la rhétorique un discours de Muret conseillant l'étude de la théologie: "ut quae digna hominis excellentia; utilis in omni vita, maxime publica [l'utile]; et honorabilis [l'honnête]». Voir aussi la péroraison, sous forme d'exhortation, d'un discours prêchant la vie de courtisan, bien digne d'un noble (p. 1037).

7. Marco-Antonio Ferrazzi (1661-1748), Exercitationes rhetoricae in praecipuas P. Virgilii Maronis orationes..., Padoue, Presses du Séminaire [G. Manfrè], 1694 ; pas de classement par types. La moitié de ses 88 analyses est sur le site de l'équipe.

8. La douleur «qui se manifeste extérieurement, d'ordinaire à l'occasion de la mort d'une personne chère » (Gaffiot).

9. Vossius, Rhetorice contracta, Leipzig, Kirchner, 1660, p.179: «DE ORATIONE, QVA DISCEDENTES vel domum redeuntes utimur, vel alium discedentem prosequimur»; Scaliger, Poetices, III, chap. 106, tout début : «Contra apobaterion carmen erit, quo discedentes utuntur » (f. 159 $\mathrm{r}^{\circ}$; éd. Deitz, p. 128 ; carmen car Scaliger décrit dans ce livre III des types de poèmes).

10. Sur Vossius, voir le résumé de Peter Mack, A History of Renaissance Rhetoric, 1380-1620, Oxford UP, 2011, p. 192-196, à partir de C. S. M. Rademaker, Life and Work of G. J. Vossius, Assen, Van Gorcum, 1981.

11. Rhetorice contracta, Leipzig, Kirchner, 1660, f. $):\left(6 v^{\circ}-7 r^{\circ}\right.$. Les mêmes listes et accolades, attribuées à "Jacob Thomas, professeur d'éloquence à l'Académie de Leipzig ", se retrouvent dans l'éd. Madrid, Anton. Sancha, 1781 (Google), p. LXVIII-LXIX : elles sont précédées du même avis au lecteur par l'éditeur qu'en 1660.

12. De conscribendis epistolis, chap. 32, LB I, 380 (c'est-à-dire : éd. de Leyde, tome I, col. 380 ; éd. Margolin, p. 311). Nous mettons tous les termes au nominatif, au lieu de "conciliationem ", etc. La liste est très différente pour l'épidictique : éloge du lieu ou « topographie », etc.

13. Là où Juste Lipse en 1591 donne une liste très abrégée de sept types de lettres (consolatio, admonitio, petitio, objurgatio, excusatio, suasio, laudatio), son commentateur de 1671 complète par une liste de près de 44 items (p. 15 et 93-95 de son Institutio, voir la Bibliographie). 
14. Cf. dans la rhétorique abrégée de Vossius, II, $23, \S 1$ : « Graeci orationes, vel epistolas adhortatorias vocant protreptikas, nouthetikas, parainetikas. »

15. Rhetorice contracta, II, 16, au tout début de ses deux remarques d'ensemble (l'inventio traitant de la matière, et la dispositio de la forme, l'Inventio specialis est chez Vossius comme une transition avec son livre III, sur la dispositio) : « $\$ 1$. Nous en venons maintenant à la seconde partie de l'Invention: laquelle est plus spécifique que la première, et moins simple. Dans le traité précédent<sur l'Invention en général>, nous avons exposé séparément les preuves et les mœurs <oratoires>. Ce traité-ci montre comment les mêmes se joignent et se mélangent à l'intérieur d'une matière particulière. $\$ 2$. Cette matière spécifique est, pour ce qui relève des passions et des mœurs, à référer aux lieux des passions et des mœurs ; pour ce qui relève des preuves, elle appartient soit au genre épidictique, soit au délibératif, soit au judiciaire. » ( $\$ 1$. A CCEDAMUS nunc ad alteram Inventionis partem : quae magis specialis est, et minus simplex. Haec docet, ut ea, quae de probationibus, moribus, seorsum antea exposuimus, in materia particulari coeant, ac misceantur. § 2. Specialis ista materies, uti, ratione affectuum morumque, pertinet ad affectuum et morum locos : ita ratione probationum, vel generis est demonstrativi, vel deliberativi, vel juridicialis.)

16. LB I, 380 (éd. Margolin, p. 310) : " Ad haec [les genres délibératif, judiciaire et épidictique] tanquam ad fontes pleraeque literarum formae referuntur ». Sebastiano Gassmayr parle de formulae, diminutif de formae, pour dire que les types de discours sont plus brefs que le grand discours d'assemblée : « il y a dans l'éloquence profane [par opposition à l'éloquence sacrée] divers petits types [formulae] de discours de congratulation, de consolation, d'admonestation, d'exhortation, [...], de supplication, d'expostulatio ", etc. (Tabulae sacrae et profanae eloquentiae, Innsbruck, Mich. Ant. Wagner, 1756, p. 331 : «In foro profano variae sunt formulae gratulatoriae, consolatoriae, admonitoriae, hortatoriae, replicatoriae, duplicatoriae, supplicatoriae, expostulatoriae, consultatoriae, etc. $»)$.

17. À propos du discours de Junon à Éole (Aeneis I, 65-75) : « sciendum est secundum hunc ordinem omnes petitiones formare Vergilium, il faut savoir que c'est selon cet ordre [ce plan] que sont formées toutes les requêtes que l'on trouve chez Virgile ». La possibilité même de généraliser à toute sorte de requête fait débat chez les divers commentateurs de Virgile aux XVI ${ }^{\mathrm{e}}$ et XVII ${ }^{\mathrm{e}}$ siècles, sensibles à ce que cette requête-ci a en fait de «tyrannique ».

\section{AUTEUR}

\section{FRANCIS GOYET}

Univ. Grenoble-Alpes, RARE - Rhétorique de l'Antiquité à la Révolution 